\title{
Exciton Binding Energies in ZnSSe/MgSSe Quantum Wells Lattice Matched to GaAs Substrates
}

\author{
Chikara Onodera* \\ Electronic Engineering Course, Aomori Prefectural Towada Technical Senior High School, \\ 215-1 Shimotai Sanbongi, Towada, Aomori 034-0001, Japan \\ Masaaki Yoshida \\ Department of Electrical and Computer Engineering, Hachinohe National College of Technology, \\ 16-1 Uwanotai, Tamonoki, Hachinohe, Aomori 039-1192, Japan
}

(Received 31 March 2011; Accepted 19 April 2011; Published 14 May 2011)

\begin{abstract}
Exciton binding energies in ZnSSe/MgSSe single quantum wells (SQWs) are calculated to study their exciton properties in detail. The heavy-hole exciton binding energies are larger than the light-hole exciton binding energies in narrow wells because the degree of confinement of the heavy-hole excitons is larger than that of the light-hole excitons in these SQWs. The heavy-hole exciton binding and heavy-hole excitonic transition energies calculated for ZnSSe/MgSSe SQWs are comparable to those measured for ZnSe/MgS quantum wells.
\end{abstract}

[DOI: $10.1380 /$ ejssnt.2011.219]

Keywords: Zinc selenide; Zinc sulfide; Magnesium sulfide; Magnesium selenide; Band offset; Exciton binding energy

\section{INTRODUCTION}

The radiative recombination of excitons in $\mathrm{ZnO}$-based quantum wells (QWs) is expected to play an important role in lasing processes [1] because the exciton binding energy in bulk $\mathrm{ZnO}$ is very large $(59 \mathrm{meV})[2]$. Ohtomo et al. measured the threshold to be less than $22 \mathrm{~kW} / \mathrm{cm}^{2}$ for the stimulated emission of excitons at room temperature (RT) in ZnO-based QWs [3].

Monolithically integrated multiwavelength laser diodes (LDs) can be used to reduce the number of optical components in recordable optical media. However, the crystalline structure of AlGaAs-based (AlGaInP-based) semiconductors used in 780-nm (650-nm) LDs differs from that of $\mathrm{ZnO}$-based semiconductors used in blue LDs. Because the crystalline structure of $\mathrm{ZnSe}$ is the same as that of $\mathrm{Al}-$ GaAs (AlGaInP), ZnSe-based blue LDs can be employed in combination with AlGaAs (AlGaInP) LDs for the fabrication of monolithically integrated multiwavelength LDs.

In the case of $\mathrm{ZnSe}$, the bulk exciton binding energy $(19 \mathrm{meV})[4]$ is less than the longitudinal optical (LO)phonon energy (31 meV) [5]; hence, exciton-LO-phonon scattering can be suppressed in ZnSe-based QWs, in which the exciton binding energy is typically enhanced by a factor of 1.5-2 because of quantum confinement. Therefore, the phenomenon of radiative recombination of excitons has been exploited to enable the use of ZnSebased QWs in the fabrication of blue LDs. In particular, ZnSe/MgS QWs have been experimentally studied for their use in the fabrication of blue optoelectronic devices [6-8]. Changes in the exciton binding energies with the well width $\left(L_{w}\right)$ have been measured for $\mathrm{ZnSe} / \mathrm{MgS}$ QWs [9].

In ZnSe-based heteroepitaxial systems, the lattice mismatch between a ZnSe-based epitaxial layer and a GaAs substrate results in a large amount of lattice defects originating from the interface $[10,11]$. The lattice defect density has to be reduced to obtain LDs with long life-

*Corresponding author: ycd1ngt@yahoo.co.jp

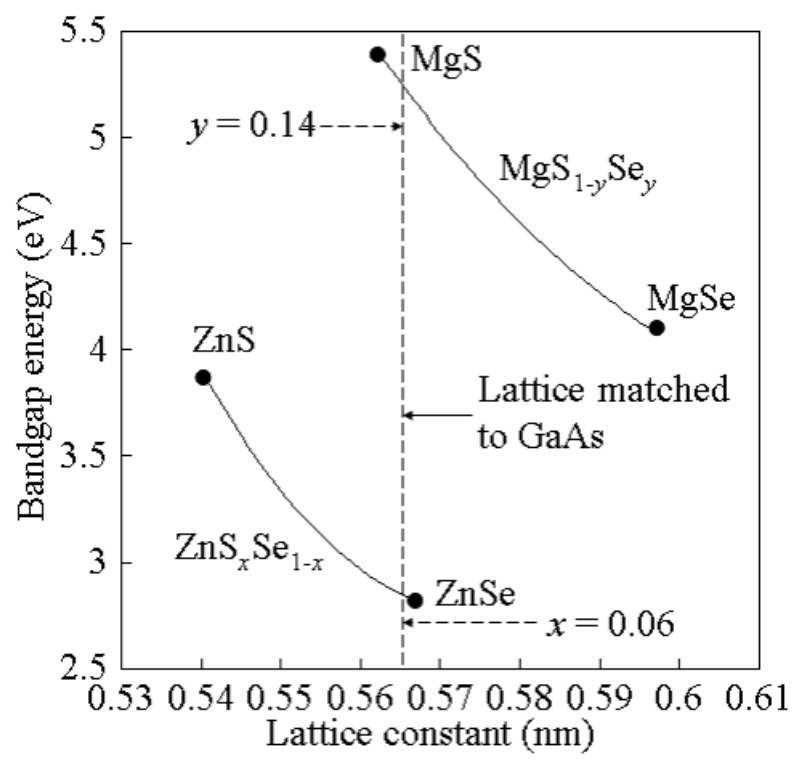

FIG. 1: Bandgap energies of II-VI semiconductors as functions of their lattice constants.

times. The ZnSe/MgS QWs have small lattice mismatch of $0.8 \%$ and are reasonably lattice matched to GaAs substrates. It was clearly observed from the transmission spectra measured for $\mathrm{ZnSe} / \mathrm{MgS}$ QWs that the transmission dip consists of the heavy- and light-hole excitons [9]. The strain owing to the lattice mismatch between $\mathrm{ZnSe}$ well and MgS barrier splits the uppermost degenerate valence band into heavy- and light-hole bands. On the other hand, in spite of the small lattice mismatch between $\mathrm{ZnSe}$ and ZnMgSSe, long-lifetime ZnSe-based LDs could not be achieved [12]. Therefore, we consider that the inhibition in lattice mismatch is important for reducing lattice defect density in ZnSe-based LDs.

The lattice constant of MgSe is larger than that of MgS, whereas the lattice constant of $\mathrm{ZnS}$ is smaller than that of ZnSe (Fig. 1). Therefore, the lattice matching between a $\mathrm{ZnS}_{x} \mathrm{Se}_{1-x}$ well (or a $\mathrm{MgS}_{1-y} \mathrm{Se}_{y}$ barrier) layer and a GaAs substrate is expected to be achieved by adjusting the sulfur $(\mathrm{S})(x)$ [or selenium $(\mathrm{Se})(y)$ ] content. 
TABLE I: Physical parameters used in the calculation.

\begin{tabular}{|c|c|c|c|c|}
\hline & $\mathrm{ZnSe}$ & $\mathrm{ZnS}$ & MgSe & $\mathrm{MgS}$ \\
\hline Lattice constant (nm) & $0.56681[15]$ & $0.54093[15]$ & $0.5964[16]$ & $0.5615[17]$ \\
\hline Bandgap energy at $4.2 \mathrm{~K}(\mathrm{eV})$ & $2.82[18]$ & $3.84[18]$ & $4.1[16]$ & $5.4[17]$ \\
\hline Top valence band energy (eV) & $-10.56[19]$ & $-11.84[19]$ & $-11.0[19]$ & $-11.93[19]$ \\
\hline Electron effective mass & $0.14[20]$ & $0.28[20]$ & & \\
\hline Luttinger parameter $\gamma_{1}$ & $2.45[21]$ & $2.54[20]$ & & \\
\hline Luttinger parameter $\gamma_{2}$ & $0.61[21]$ & $0.75[20]$ & & \\
\hline Relative dielectric constant & $8.8[14]$ & $8.6[22]$ & & \\
\hline Bowing parameter of $\mathrm{ZnSSe}$ & $0.63[23]$ & & & \\
\hline Bowing parameter of MgSSe & $0.4911[23]$ & & & \\
\hline
\end{tabular}

When $x=0.06$ and $y=0.14, \mathrm{ZnS}_{x} \mathrm{Se}_{1-x} / \mathrm{MgS}_{1-y} \mathrm{Se}_{y}$ single QWs (SQWs) can be lattice matched to GaAs substrates. The bandgap energy of the $\mathrm{MgS}_{0.86} \mathrm{Se}_{0.14}$ layer is larger than that of the $\mathrm{ZnS}_{0.06} \mathrm{Se}_{0.94}$ layer (Fig. 1). The difference between the bandgaps of the $\mathrm{ZnS}_{0.06} \mathrm{Se}_{0.94}$ and $\mathrm{MgS}_{0.86} \mathrm{Se}_{0.14}$ layers is smaller than that of the $\mathrm{MgS}$ and ZnSe layers. However, this difference between the two sets of layers is small. It is expected that the exciton binding energies in the $\mathrm{ZnS}_{0.06} \mathrm{Se}_{0.94} / \mathrm{MgS}_{0.86} \mathrm{Se}_{0.14}$ SQWs are comparable to that in the ZnSe/MgS SQWs. The $\mathrm{ZnS}_{0.06} \mathrm{Se}_{0.94} / \mathrm{MgS}_{0.86} \mathrm{Se}_{0.14} \mathrm{SQWs}$ are therefore an important candidate for use in the fabrication of blue LDs on GaAs substrates. Estimation of the exciton binding energies in $\mathrm{ZnS}_{0.06} \mathrm{Se}_{0.94} / \mathrm{MgS}_{0.86} \mathrm{Se}_{0.14} \mathrm{SQWs}$ is necessary for tuning the optical properties of these QWs for various LD applications.

In this study, we calculate the heavy- and lighthole exciton binding energies as functions of the $L_{w}$ in $\mathrm{ZnS}_{0.06} \mathrm{Se}_{0.94} / \mathrm{MgS}_{0.86} \mathrm{Se}_{0.14} \mathrm{SQWs}$. We follow a variational approach. Moreover, we calculate the exciton transition energies to achieve appropriate adjustment of the optical bandgap for various applications.

\section{CALCULATION METHODS}

Greene et al. have reported the details of the method for calculating binding energies in QWs [13]. We use a variational method for calculating the exciton binding energies. The exciton Hamiltonian is given by [13]

$$
\begin{aligned}
H=- & \frac{\hbar^{2}}{2 \mu_{ \pm}} \frac{1}{r} \frac{\partial}{\partial r} r \frac{\partial}{\partial r}-\frac{\hbar^{2}}{2 m_{e}^{*}} \frac{\partial^{2}}{\partial z_{e}^{2}}-\frac{\hbar^{2}}{2 m_{ \pm}} \frac{\partial^{2}}{\partial z_{h}^{2}} \\
& -\frac{e^{2}}{\epsilon \sqrt{r^{2}+\left(z_{e}-z_{h}\right)^{2}}} \\
& +V_{e}\left(z_{e}\right)+V_{h}\left(z_{h}\right),
\end{aligned}
$$

where $\mu_{ \pm}$is the exciton reduced mass and $\epsilon$, the dielectric constant. $V_{e}\left(z_{e}\right)$ and $V_{h}\left(z_{h}\right)$ are the conduction and valence band offsets, respectively. A trial function [14] is given by

$$
\varphi=\phi_{e}\left(z_{e}\right) \phi_{h}\left(z_{h}\right) \exp \left[-\alpha\left\{r^{2}+\beta\left(z_{e}-z_{h}\right)^{2}\right\}^{1 / 2}\right],
$$

where $\phi_{e}\left(z_{e}\right)$ and $\phi_{h}\left(z_{h}\right)$ are the electron and heavy-hole wave functions, respectively. $\alpha$ and $\beta$ are variational parameters.

The energy at the bottom of the conduction band in $\mathrm{ZnS}_{x} \mathrm{Se}_{1-x}$ is calculated by linear interpolation without using a bowing parameter. On the other hand, the energy at the top of the valence band in $\mathrm{ZnS}_{x} \mathrm{Se}_{1-x}$ is calculated by linear interpolation using a bowing parameter. In $\mathrm{ZnS}_{x} \mathrm{Se}_{1-x}$, the anions ( $\mathrm{S}$ and $\mathrm{Se}$ ) have a strong influence on the valence band, whereas $\mathrm{Zn}$ cations determine the energy at the bottom of the conduction band. The energies at the bottom of the conduction band and the top of the valence band in $\mathrm{MgS}_{1-y} \mathrm{Se}_{y}$ are calculated in the same manner.

The valence band offset is calculated as the difference between the energy at the top of the valence band in the well and that in the barrier. The conduction band offset is calculated as the difference between the energy at the bottom of the conduction band in the well and that in the barrier.

The heavy-hole exciton transition energy is determined by subtracting the heavy-hole exciton binding energy from the effective heavy-hole bandgap energy; the effective heavy-hole bandgap energy is calculated as the summation of the minimum conduction subband energy, minimum heavy-hole subband energy, and heavy-hole bandgap energy of the well layer. The light-hole exciton transition energy is calculated in the same manner.

\section{RESULTS AND DISCUSSION}

The physical parameters used in our calculation are listed in Table I. The parameters pertaining to the alloy material are derived by linear interpolation. The effective masses of heavy and light holes in the $z$-direction and for the $x-y$ plane are determined in terms of the well-known Luttinger parameters. For simplification of our calculation, the effective masses of electrons, heavy holes, and light holes are assumed to be position independent and equal to the effective masses of those of $\mathrm{ZnS}_{0.06} \mathrm{Se}_{0.94}$. Conduction and valence band offsets are estimated to be $1525 \mathrm{meV}$ and $788 \mathrm{meV}$, respectively.

Figure 2 shows heavy- and light-hole exciton binding energies in $\mathrm{ZnS}_{0.06} \mathrm{Se}_{0.94} / \mathrm{MgS}_{0.14} \mathrm{Se}_{0.86} \mathrm{SQWs}$ as functions of $L_{w}$. For comparison, the heavy- and light-hole exciton binding energies measured for $\mathrm{ZnSe} / \mathrm{MgS}$ multiple QWs (MQWs) [9] are also plotted. The heavyhole exciton binding energy measured for a well width of $5 \mathrm{~nm}$ is considerably larger than that calculated for $\mathrm{ZnS}_{0.06} \mathrm{Se}_{0.94} / \mathrm{MgS}_{0.14} \mathrm{Se}_{0.86}$ SQWs. The measured heavy-hole exciton binding energies show marked decreases when the value of $L_{w}$ increases from $5 \mathrm{~nm}$ to $10 \mathrm{~nm}$ because of a decrease in the quantum confinement effect 


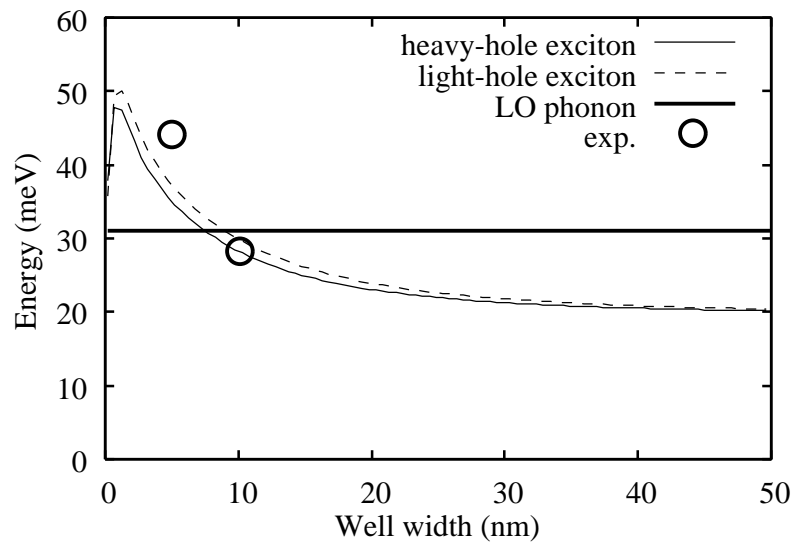

FIG. 2: Heavy- and light-hole exciton binding energies calculated for $\mathrm{ZnS}_{0.06} \mathrm{Se}_{0.94} / \mathrm{MgS}_{0.86} \mathrm{Se}_{0.14} \mathrm{SQWs}$ as functions of $L_{w}$. Solid and dashed curves indicate the plots of heavy- and light-hole exciton binding energies, respectively. The bold line indicates the plot of LO-phonon energy of ZnSe. Open circles indicate the heavy-hole exciton binding energies measured for ZnSe/MgS SQWs reported by Urbaszek et al. [9].

on excitons. On the other hand, the heavy-hole exciton binding energy measured for a well width of $10 \mathrm{~nm}$ is close to that calculated for $\mathrm{ZnS}_{0.06} \mathrm{Se}_{0.94} / \mathrm{MgS}_{0.14} \mathrm{Se}_{0.86}$ SQWs. The heavy-hole exciton binding energies calculated for $\mathrm{ZnS}_{0.06} \mathrm{Se}_{0.94} / \mathrm{MgS}_{0.14} \mathrm{Se}_{0.86} \mathrm{SQWs}$ are comparable to those measured for $\mathrm{ZnSe} / \mathrm{MgS}$ MQWs [9]. To take a first step in designing ZnSSe/MgSSe QWs, we calculate the exciton binding energy using static dielectric constants for the ZnSSe well and MgSSe barrier. Senger and Bajaj reported that the exciton binding energies calculated for $\mathrm{ZnSe} / \mathrm{MgS}$ QWs by considering the Pollmann and Büttner (PB) potential are larger than those calculated by ignoring the PB potential [24]. The exciton binding energies calculated for $\mathrm{ZnSe} / \mathrm{MgS} \mathrm{QWs}$ by considering the $\mathrm{PB}$ potential agree rather well with those measured for for $\mathrm{ZnSe} / \mathrm{MgS} \mathrm{QWs}$ [24]. For simplification of our calculation, we neglect the effect of $\mathrm{PB}$ potential in this study. For more realistic understanding of the changes in the exciton binding energies with $L_{w}$, the calculation of the exciton binding energy using $\mathrm{PB}$ potential is necessary.

With a decrease in the value of $L_{w}$, the heavy- and light-hole exciton binding energies increase to reach a maximum value and then decrease rapidly. This is because when the value of $L_{w}$ decreases, the exciton wave function is compressed in the wells and extends to the barrier region [13]. The shape of the curve representing the dependence of the heavy- and light-hole exciton binding energies on the $L_{w}$ is consistent with that reported by Greene et al. [12]. Changes in the binding energies of the heavy- and light-hole excitons with $L_{w}$ are essentially similar.

The maximum heavy-hole (light-hole) exciton binding energy is $50.3 \mathrm{meV}$ (47.9 meV). The binding energy of the heavy-hole excitons calculated by neglecting the quantum confinement effect $(18.1 \mathrm{meV})$ is smaller than that of the light-hole excitons calculated by neglecting the quantum confinement effect $(20.7 \mathrm{meV})$. However, the maximum binding energies of the heavy-hole excitons calculated by considering the quantum confinement effect are

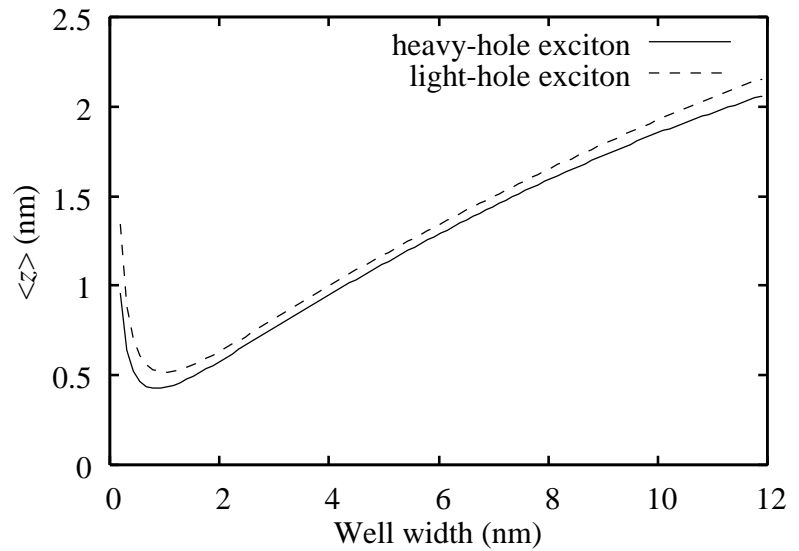

FIG. 3: Spatial separation of heavy- and light-hole excitons in the $z$-direction $(\langle z\rangle)$ in $\mathrm{ZnS}_{0.06} \mathrm{Se}_{0.94} / \mathrm{MgS}_{0.86} \mathrm{Se}_{0.14} \mathrm{SQWs}$ as functions of $L_{w}$. Solid and dashed curves indicate the plots of $\langle z\rangle$ for heavy- and light-hole excitons, respectively.

larger than those of the light-hole excitons calculated under the same consideration. This is because the degree of compression of the exciton wave function for a heavyhole exciton is larger than that for a light-hole exciton. In order to determine the degree of compression of the exciton wave function, we calculate the dependence of spatial separation of excitons in the $z$-direction $(\langle z\rangle)$ on $L_{w} \cdot\langle z\rangle$ is given as [25]

$$
\langle z\rangle=\left\langle\varphi\left|\left(z_{e}-z_{h}\right)^{2}\right| \varphi\right\rangle^{1 / 2},
$$

where $\varphi$ is the trial function. The calculated results are shown in Fig. 3. $\langle z\rangle$ is minimum at small $L_{w}$ and increases with $L_{w}$. The value of $\langle z\rangle$ for the heavy-hole excitons is smaller than that for the light-hole excitons at the same $L_{w}$. The minimum $\langle z\rangle$ for the heavy-hole excitons is smaller than that for the light-hole excitons; hence, the larger degree of compression for the heavy-hole excitons is larger than that for the light-hole excitons.

Moreover, to determine the degree of compression of the exciton wave function, the $L_{w}$ dependence of the probability of finding an exciton inside the well $(P)$ is calculated. $P$ is given as [13]

$$
P=\int_{0}^{\infty} \int_{-L_{w} / 2}^{L_{w} / 2} \int_{-L_{w} / 2}^{L_{w} / 2}|\varphi|^{2} d z_{e} d z_{h} r d r .
$$

The calculated results are shown in Fig. 4. $P$ increases with $L_{w}$ and is almost equal to 1 . The value of $P$ for the heavy-hole excitons is larger than that for the lighthole excitons at the same $L_{w}$. The $L_{w}$ dependence of $P$ suggests that the degree of confinement of the heavy-hole excitons is larger than that of the light-hole excitons.

When the value of $L_{w}$ for the $\mathrm{ZnS}_{0.06} \mathrm{Se}_{0.94} /$ $\mathrm{MgS}_{0.14} \mathrm{Se}_{0.86} \mathrm{SQWs}$ is in the range of $0.2-8.0 \mathrm{~nm}(0.2-$ $9.0 \mathrm{~nm}$ ), the heavy-hole (light-hole) exciton binding energies are all larger than the LO-phonon energy of ZnSe $(31 \mathrm{meV})$ [5]. This indicates that the exciton characteristics are predominant even at RT. The strong interaction between the excitons and LO phonons usually results in the dissociation of the excitons at higher temperatures [9]. Ionization of excitons should be prevented in a system in which all the excitations of the exciton have an energy 


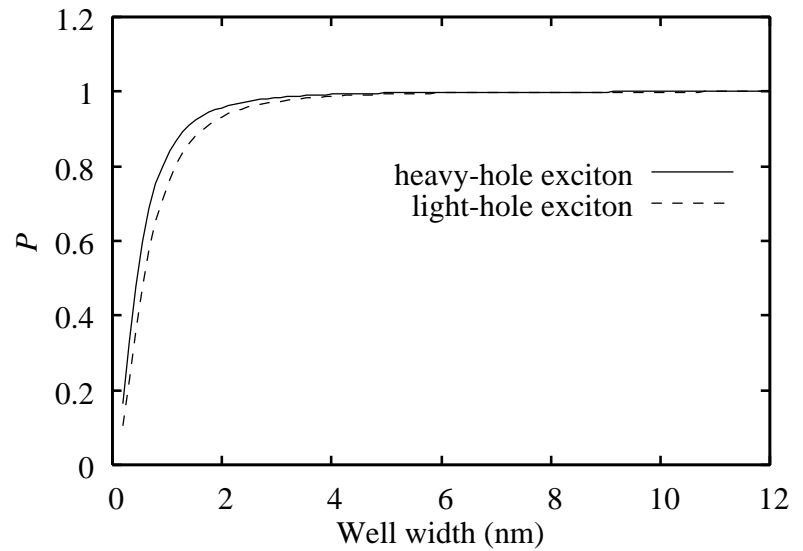

FIG. 4: Probability of finding heavy- and light-hole excitons inside the $\mathrm{ZnS}_{0.06} \mathrm{Se}_{0.94}$ well layer $(P)$ as functions of $L_{w}$. Solid and dashed curves indicate the plots of $P$ for heavy- and lighthole excitons, respectively.

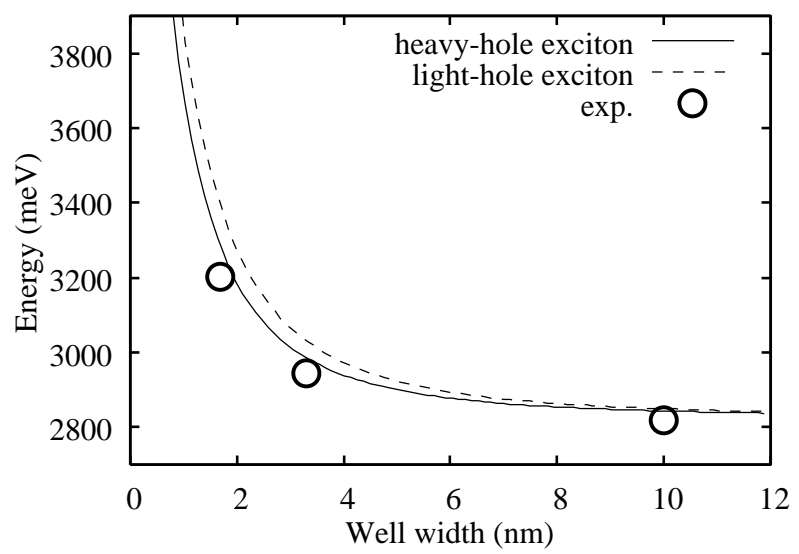

FIG. 5: Calculated heavy- and light-hole exciton transition energies in $\mathrm{ZnS}_{0.06} \mathrm{Se}_{0.94} / \mathrm{MgS}_{0.86} \mathrm{Se}_{0.14} \mathrm{SQWs}$ as functions of $L_{w}$. Solid and dashed curves indicate the plots of heavy- and light-hole exciton transition energies, respectively. Open circles indicate the photoluminescence (PL) peak energies measured for ZnSe/MgS SQWs reported by Urbaszek et al. [9]. larger than that of the LO phonon [9]. For a more realistic understanding of the exciton stability, the calculation of the excited exciton states is necessary. For simplification of our calculation, we limit our discussion to the binding energy of the ground exciton state; therefore, our result does not provide a complete picture of the exciton stability.

For comparison, we also calculate the heavyand light-hole exciton transition energies in $\mathrm{ZnS}_{0.06} \mathrm{Se}_{0.94} / \mathrm{MgS}_{0.86} \mathrm{Se}_{0.14} \mathrm{SQWs}$ as a function of $L_{w}$. The calculated results are plotted in Fig. 5, where open circles indicate the photoluminescence (PL) peak energies measured for $\mathrm{ZnSe} / \mathrm{MgS}$ SQWs presented in ref. 9. The heavy- and light-hole exciton transition energies calculated by us are larger than the PL peak energies measured for $\mathrm{ZnSe} / \mathrm{MgS}$ SQWs [9]. This is because the bandgap energy of $\mathrm{ZnS}_{0.06} \mathrm{Se}_{0.94}$ is larger than that of ZnSe. The difference between the calculated and measured values is small.

\section{CONCLUSION}

We have calculated the binding energies of heavy- and light-hole excitons in $\mathrm{ZnS}_{0.06} \mathrm{Se}_{0.94} / \mathrm{MgS}_{0.86} \mathrm{Se}_{0.14} \mathrm{SQWs}$. The maximum binding energy of the heavy-hole (lighthole) exciton is $50.3 \mathrm{meV}(47.9 \mathrm{meV})$. The $L_{w}$ dependence of $\langle z\rangle$ and $P$ suggests that the degree of confinement of the heavy-hole excitons is larger than that of the light-hole excitons. When the value of $L_{w}$ is in the range of $0.2-8.0 \mathrm{~nm}$ $(0.2-9.0 \mathrm{~nm})$, the heavy-hole (light-hole) exciton binding energies are all larger than the LO-phonon energy of ZnSe (31 meV). The heavy- and light-hole exciton transition energies calculated for $\mathrm{ZnS}_{0.06} \mathrm{Se}_{0.94} / \mathrm{MgS}_{0.86} \mathrm{Se}_{0.14} \mathrm{SQWs}$ are comparable to the $\mathrm{PL}$ peak energies measured for ZnSe/MgS QWs. Therefore, $\mathrm{ZnS}_{0.06} \mathrm{Se}_{0.94} / \mathrm{MgS}_{0.86} \mathrm{Se}_{0.14}$ SQWs can possibly be used in the fabrication of blue laser devices using exciton emission at RT.
[1] R. Cingolani, L. Calcagnile, G. Coli, R. Rinaldi, M. Lomascolo, M. DiDio, A. Franciosi, L. Vanzetti, G. C. LaRocca, and D. Campi, J. Opt. Soc. Am. B13, 1268 (1996).

[2] S. Shigemori, A. Nakamura, J. Ishihara, T. Aoki, and J. Temmyo, Jpn. J. Appl. Phys. 43, L1088 (2004).

[3] A. Ohtomo, K. Tamura, M. Kawasaki, T. Makino, Y. Segawa, Z. K. Tang, G. K. L. Wong, Y. Matsumoto, and H. Koinuma, Appl. Phys. Lett. 77, 2204 (2000).

[4] M. Isshiki, T. Toga, K. Masumoto, K. Mochizuki, and W. Uchida, Jpn. J. Appl. Phys. 30, 623 (1991).

[5] T. Miyajima, F. P. Logue, J. F. Donegan, J. Hegarty, H. Okuyama, A. Ishibashi, and Y. Mori, Appl. Phys. Lett. 66, 180 (1995).

[6] H. Suzuki, T. Obinata, H. Nashiki, K. Uesugi, and I. Suemune, Jpn. J. Appl. Phys. 35, L1658 (1996).

[7] H. Nashiki, I. Suemune, H. Suzuki, and K. Uesugi, Jpn. J. Appl. Phys. 36, 4199 (1997).

[8] H. Kumano, H. Nashiki, I. Suemune, H. Suzuki, K. Uesugi, A-Q. He, N. Otsuka, Jpn. J. Appl. Phys. 39, 501 (2000).

[9] B. Urbaszek, C. Morhain, C. Bradford, C. B. O'Donnell,
S. A. Telfer, X. Tang, A. Balocchi, K. A. Prior, B. C. Cavenett, C. M. Townsley, and R. J. Nicholas, J. Phys.: Condens. Matter 13, 2317 (2001).

[10] T. Narusawa, F. Nishiyama, Z. Zhu, and T. Yao, Jpn. J. Appl. Phys. 36, L12 (1997).

[11] H. Wenisch, M. Behringer, M. Fehrer, M. Klude, A. Isemann, K. Ohkawa, and D. Hommel, Jpn. J. Appl. Phys. 38, 2590 (1999).

[12] H. Wenish, M. Behringer, M. Fehrer, M. Klude, A. Isemann, K. Okawa, and D. Hommel. Jpn. J. Appl. Phys. 38, 2590 (1999).

[13] R. L. Greene, K. K. Bajaj, and D. E. Phelps, Phys. Rev. B 29, 1807 (1984).

[14] R. T. Senger and K. K. Bajaj, Phys. Rev. B 68, 205314 (2003).

[15] K. Ichino, Y. Kawakami, Sz. Fujita, and Sg. Fujita, Oyo Butsuri 61, 117 (1992) [in Japanese].

[16] D. Wolverson, D. M. Bird, C. Bradford, K. A. Prior, and B. C. Cavenett, Phys. Rev. B 64, 113203 (2001).

[17] J. Puls, M. Rade, A. Siarkos, and F. Henneberger, Phys. Rev. B 57, 14749 (1998).

[18] Y. Wu, K. Ichino, Y. Kawakami, Sz. Fujita, and Sg. Fujita, 
Jpn. J. Appl. Phys. 31, 1737 (1992).

[19] W. A. Harrison, Elementary Electronic Structures Revised Edition (World Scientific, Singapore, 2004). The top of the valence band energies of $\mathrm{ZnSe}$ and $\mathrm{ZnS}$ are calculated by taking the effect of d-states into account.

[20] P. Lawaetz, Phys. Rev. B 4, 3460 (1971).

[21] A. Nardis, V. Pellegrini, R. Colombelli, F. Beltram, L. Vanzetti, A. Franciosi, I. N. Krivorotov, and K. K. Bajaj, Phys. Rev. B 61, 1700 (2000).
[22] D. A. Berlincourt, H. Jaffe, and L. R. Shiozawa, Phys. Rev. 129, 1009 (1963).

[23] M. Rabah, B. Abbar, Y. Al-Douri, B. Bouhafs, and B. Sahraoui, Materials Sci. and Eng. B 100, 163 (2003).

[24] R. T. Senger and K. K. Bajaj, Phys. stat. sol. (b) 241, 1896 (2004).

[25] Y. Shinozuka and M. Matsuura, Phys. Rev. B 28, 4878 (1983) [Errata; 29, 3717 (1984)]. 